\title{
Semileptonic and leptonic $D$ decays at BESIII
}

\author{
Ke Liu (For BESIII Collaboration)* \\ Central China Normal University, 430079,P.R. China \\ E-mail: liuke@ihep.ac.cn
}

Using the world's largest $e^{+} e^{-}$collision data samples taken at $\sqrt{s}=3.773 \mathrm{GeV}$ and $\sqrt{s}=4.178$ $\mathrm{GeV}$ with the integrated luminosities $2.93 \mathrm{fb}^{-1}$ and $3.19 \mathrm{fb}^{-1}$ accumulated by the BESIII detector at the Beijing Electron Positron Collider, precise measurements of the branching fractions of $D \rightarrow K \mu^{+} v_{\mu}, D \rightarrow \pi \mu^{+} v_{\mu}$ and $D_{(s)}^{+} \rightarrow \mu^{+} v_{\mu}$ are reported. Combining the known branching fractions of $D \rightarrow \bar{K} e v_{e}, D \rightarrow \pi e v_{e}$ and $D_{(s)}^{+} \rightarrow \tau^{+} v_{\tau}$, we have tested the lepton flavor universality with $D \rightarrow \bar{K} \ell^{+} v_{\ell}, D \rightarrow \pi \ell^{+} v_{\ell}$ and $D_{(s)}^{+} \rightarrow \ell^{+} v_{\ell}$ decays. We also determine the CKM matrix element $\left|V_{c s(d)}\right|$, the form factors $f_{+}^{D \rightarrow K(\pi)}(0)$ and $f_{+}^{D \rightarrow \eta^{\prime}(\eta)}(0)$ of semileptonic $D$ decays, and the $D_{(s)}^{+}$decay constant $f_{D_{(s)}^{+}}$with the most precise accuracies to date. Furthermore, searches for semileptonic $D$ decays to scalar and axial-vector mesons are also performed, and these studies help us understand the internal structure of light mesons.

The 29th International Symposium on Lepton Photon Interactions at High Energies - LeptonPhoton2019 August 5-10, 2019

Toronto, Canada

\footnotetext{
* Speaker.

${ }^{\dagger}$ I would like to thank for the support of the Joint Funds of the National Natural Science Foundation of China (Grants No.U1832107).
} 


\section{Introduction}

In the standard model (SM), the strong and weak interactions in leptonic and semileptonic $D$ decays can be well separated, as shown in Fig. $1 . D_{(s)}^{+}$mesons decay into $\ell \boldsymbol{v}_{\ell}$ via a virtual $W^{+}$ boson and the decay rate can be parameterized by the $D_{(s)}^{+}$decay constant $f_{D_{(s)}}^{+}$via

$$
\Gamma\left(D \rightarrow \ell v_{\ell}\right)=\frac{G_{F}^{2}}{8 \pi}\left(\left|V_{c s(d)}\right| f_{D_{(s)}^{+}}\right)^{2} m_{\ell}^{2} m_{D}\left(1-\frac{m_{\ell}^{2}}{m_{D_{(s)}^{+}}^{2}}\right)^{2}
$$

where $f_{D_{(s)}^{+}}$is the $D$ meson decay constant, $V_{c s(d)}$ is the Cabbibo-Kobayashi-Maskawa (CKM) matrix element describing the mixing between quark weak eigenstates and flavor eigenstates, $G_{F}$ is the Fermi coupling constant, $m_{D_{(s)}^{+}}$and $m_{\ell}$ are the masses of $D$ meson and lepton [1]. And semileptonic $D$ decay rate can be parameterized by the quark mixing matrix element and the form factor of hadronic weak current in theory. Setting the simplest case, for $D \rightarrow K(\pi) e^{+} v_{e}$, it's differential decay rate can be simply written as

$$
\frac{d \Gamma\left(D \rightarrow K(\pi) e^{+} v_{e}\right)}{d q^{2}}=\frac{G_{F}^{2}}{24 \pi^{3}}\left(\left|V_{c s(d)}\right| f_{+}^{K(\pi)}\left(q^{2}\right)\right)^{2}\left|\vec{p}_{K(\pi)}\right|^{3},
$$

where $f_{+}^{K(\pi)}\left(q^{2}\right)$ are the $D$ meson decay constant and hadronic form factor and $\vec{p}_{K(\pi)}$ is the momentum of the pseudoscalar meson.
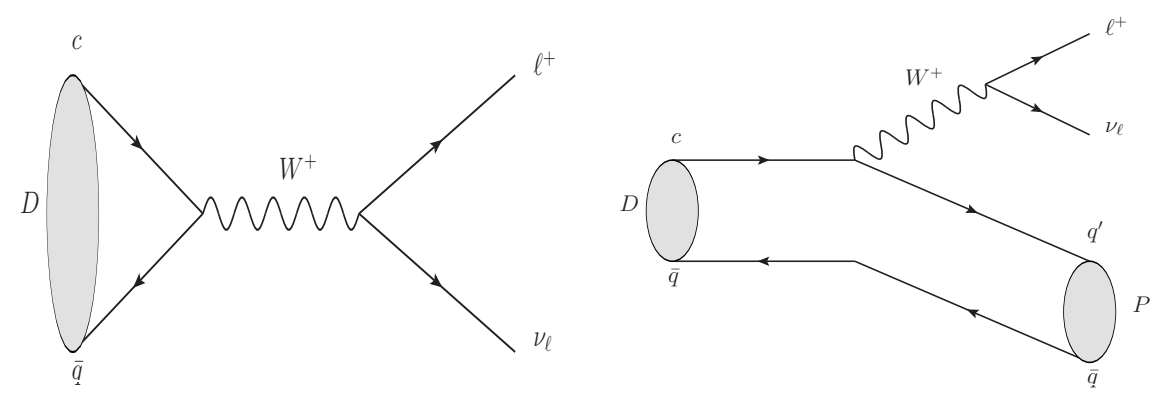

Figure 1: Feynman diagrams for leptonic $D$ decays (left) and semileptonic $D$ decays to pseudoscalar mesons (right).

From experimental studies of semileptonic and purely leptonic decays of charmed mesons, one can determine the decay constants $f_{D_{(s)}^{+}}$, or the form factors and the CKM matrix elements $\left|V_{c s(d)}\right|$, which are important to calibrate the theoretical calculations and the CKM matrix unitarity test. For leptonic $D$ decays and semileptonic $D$ decays to pseudoscalar mesons, the hadronic currents are almost cancelled out when we calculate the ratio of decay rates to different generations of leptons. With the precisely determined SM prediction, we can test for lepton flavor universality (LFU) in the charm sector at a very good precision.

Measurements of leptonic and semileptonic charm decays are mainly carried out in the charm factory, nowadays mostly at the BESIII detector [2] with $2.93 \mathrm{fb}^{-1}$ and $3.19 \mathrm{fb}^{-1}$ data collected at center mass energy $3.773 \mathrm{GeV}$ and $4.178 \mathrm{GeV}$, respectively. For charm factories which work at the energy of the $D \bar{D}$ threshold, the $D$ mesons are produced in pairs. If we first fully reconstruct a $D$ meson using hadronic decays, the other $D$ meson is then guaranteed to exist in the recoiling system. 


\section{Recent results at BESIII}

\subsection{Leptonic $D_{(s)}^{+}$decay}

BESIII has recently reported an improved measurement of $D_{s}^{+} \rightarrow \mu^{+} v_{\mu}$ decay [3] with a total of about $3.9 \times 10^{5}$ single tagged $D_{s}$ mesons reconstructed based on $3.19 \mathrm{fb}^{-1}$ data taken at a center mass energy of $4.178 \mathrm{GeV}$. Figure 2 shows the missing mass square of the neutrino, which is defined as $M_{\text {miss }}^{2}=\sqrt{E_{\text {miss }}^{2}-p_{\text {miss }}^{2}}$, where $E_{\text {miss }}$ and $p_{\text {miss }}$ are the missing energy and momentum of the candidate event. The $\mathrm{BF}$ is measured to be $\mathscr{B}\left(D_{s}^{+} \rightarrow \mu^{+} v_{\mu}\right)=(5.49 \pm 0.16 \pm 0.15) \times 10^{-3}$. Given the lifetime of the $D_{s}^{+}$meson [1], we obtain $f_{D_{s}^{+}}\left|V_{c s}\right|=246.2 \pm 3.6 \pm 3.5 \mathrm{MeV}$. If we input the

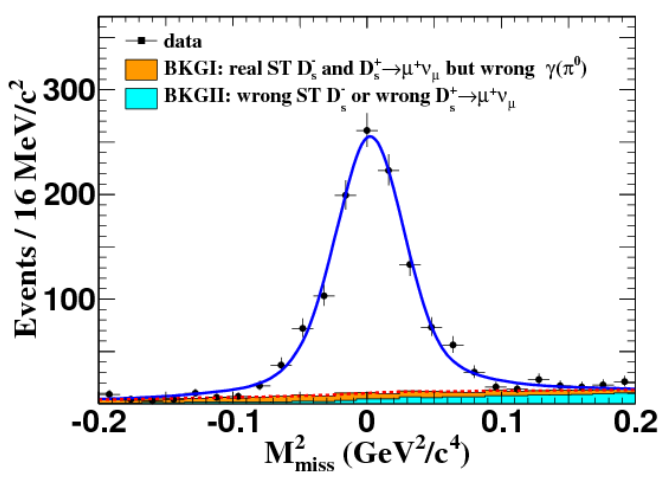

Figure 2: Fit to the $M_{\text {miss }}^{2}$ distribution of $D_{s}^{+} \rightarrow \mu^{+} v_{\mu}$ candidates, where the dots with error bars are data, the blue solid curve shows the best fit and the red dashed curve shows the background shape.

CKM matrix element $\left|V_{c s}\right|$ from a global fit [1], we have $f_{D_{s}^{+}}=252.9 \pm 3.7 \pm 3.5 \mathrm{MeV}$. In contrast, inputting $f_{D_{s}^{+}}$from Lattice QCD calculation [4, 5] gives $\left|V_{c s}\right|=0.985 \pm 0.014 \pm 0.014$. These are the most precise single measurements to date. Averaging this measurement of $\mathscr{B}\left(D_{s}^{+} \rightarrow \mu^{+} v_{\mu}\right)$ with the previous measurements $[6,7,8,9]$ and combining the world average of $\mathscr{B}\left(D_{s}^{+} \rightarrow \tau^{+} v_{\tau}\right)$ [1], we find $\frac{\mathscr{B}_{D_{s}^{+} \rightarrow \tau^{+} v_{\tau}}}{\mathscr{B}_{D_{s}^{+} \rightarrow \mu^{+} v_{\mu}}}=9.98 \pm 0.52$, which is consistent with the SM prediction of 9.74.

BESIII has also searched for $D^{+} \rightarrow \tau^{+} v_{\tau}$ with $\tau^{+} \rightarrow \pi^{+} v_{\tau}$ based on $2.93 \mathrm{fb}^{-1}$ data taken at the center mass energy of $3.773 \mathrm{GeV}$ [10], with a total of about $1.7 \times 10^{6}$ single tagged $D$ mesons reconstructed(See Fig. 3). The $D^{+} \rightarrow \tau^{+} v_{\tau}$ decay is observed for the first time with a statistical significance $>5.1 \sigma$. Combined with the previous measurement of the $\mathrm{BF}$ of $D^{+} \rightarrow \mu^{+} v_{\mu}$ from BESIII [11], we obtain $\frac{\mathscr{B}_{D^{+} \rightarrow \tau^{+} v_{\tau}}}{\mathscr{B}_{D^{+} \rightarrow \mu^{+} v_{\mu}}}=3.21 \pm 0.64_{\text {stat. }} \pm 0.43_{\text {syst. }}$ [10], which is also consistent with the SM prediction of 2.66 .

\subsection{Semileptonic $D$ decays}

In recent years, semileptonic $D$ decays to pseudoscalar mesons have been performed at BESIII for $D \rightarrow K(\pi) e^{+} v_{e}, D \rightarrow K(\pi) \mu^{+} v_{\mu}$, and $D \rightarrow \eta^{(\prime)} e^{+} v_{e}$, etc $[12,13,14,15,16,17,18]$. The measured BFs and the product of form factors at $q^{2}=0$ and CKM matrix elements are summarized in Table 1. Most of the measurements are consistent with LQCD calculations. We also find that $\frac{f_{+}^{D_{s}^{+} \rightarrow K^{0}}(0)}{f_{+}^{D^{+} \rightarrow \pi^{0}}(0)}=1.16 \pm 0.14 \pm 0.02$, which is consistent with U-spin symmetry. 

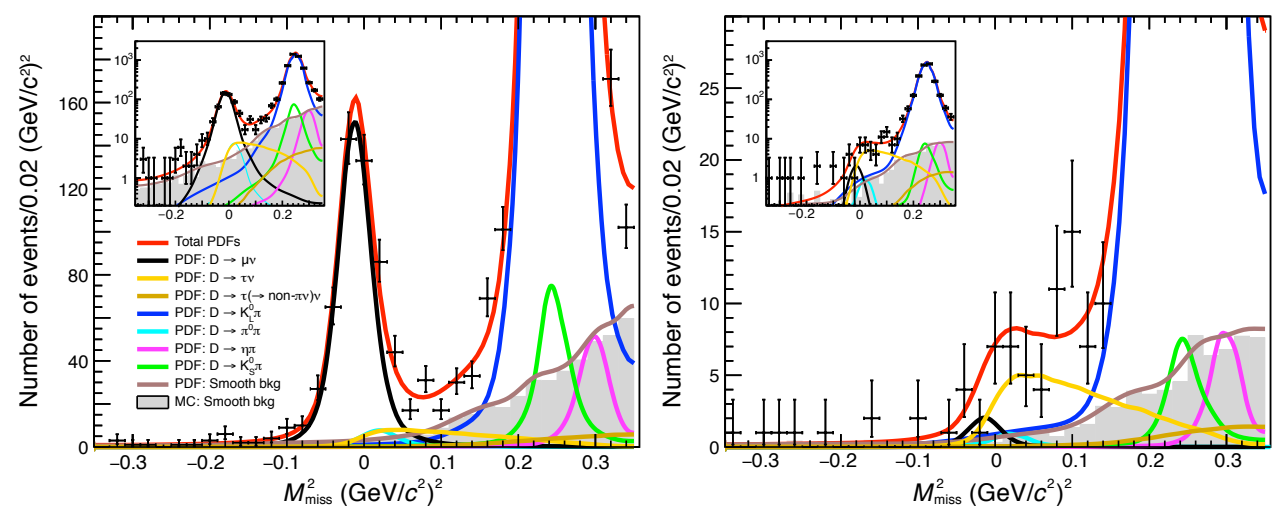

Figure 3: The simultaneous fit to the $M_{\text {miss }}^{2}$ distributions of the muon-like sample (with deposited energy in the EMC less than $300 \mathrm{MeV}$ ) and the pion-like sample (with deposited energy in the EMC larger than 300 $\mathrm{MeV})$.

Table 1: The measured BFs and product of form factors at $q^{2}=0$ and CKM matrix elements for some semileptonic $D$ decays to psuedoscalar mesons.

\begin{tabular}{cc|cc}
$\mathscr{B}\left(D^{0} \rightarrow K^{-} e^{+} v_{e}\right)$ & $(3.505 \pm 0.014 \pm 0.033) \%$ & $f_{+}^{D^{0} \rightarrow K^{-}}(0)\left|V_{c s}\right|$ & $0.7172 \pm 0.0025 \pm 0.0035$ \\
$\mathscr{B}\left(D^{0} \rightarrow K^{-} \mu^{+} v_{\mu}\right)$ & $(3.431 \pm 0.019 \pm 0.035) \%$ & $f_{+}^{D^{0} \rightarrow K^{-}}(0)\left|V_{c s}\right|$ & $0.7133 \pm 0.0038 \pm 0.0030$ \\
$\mathscr{B}\left(D^{+} \rightarrow \bar{K}^{0} e^{+} v_{e}\right)$ & $(8.60 \pm 0.06 \pm 0.015) \%$ & $f_{+}^{D^{+} \rightarrow \bar{K}^{0}}(0)\left|V_{c s}\right|$ & $0.7053 \pm 0.0040 \pm 0.0112$ \\
$\mathscr{B}\left(D_{s}^{+} \rightarrow K^{0} e^{+} v_{e}\right)$ & $(3.25 \pm 0.38 \pm 0.16) \times 10^{-3}$ & $f_{+}^{D_{s}^{+} \rightarrow K^{0}}(0)\left|V_{c d}\right|$ & $0.162 \pm 0.019 \pm 0.003$ \\
$\mathscr{B}\left(D^{0} \rightarrow \pi^{-} e^{+} v_{e}\right)$ & $(2.95 \pm 0.04 \pm 0.03) \times 10^{-3}$ & $f_{+}^{D^{0} \rightarrow \pi^{-}}(0)\left|V_{c d}\right|$ & $0.1435 \pm 0.0018 \pm 0.0009$ \\
$\mathscr{B}\left(D^{+} \rightarrow \pi^{0} e^{+} v_{e}\right)$ & $(3.63 \pm 0.08 \pm 0.05) \times 10^{-3}$ & $f_{+}^{D^{+} \rightarrow \pi^{0}}(0)\left|V_{c d}\right|$ & $0.1400 \pm 0.0026 \pm 0.0007$ \\
$\mathscr{B}\left(D^{+} \rightarrow \eta e^{+} v_{e}\right)$ & $(10.74 \pm 0.81 \pm 0.51) \times 10^{-4}$ & $f_{+}^{D^{+} \rightarrow \eta}(0)\left|V_{c d}\right|$ & $0.0786 \pm 0.0064 \pm 0.0021$ \\
$\mathscr{B}\left(D_{s}^{+} \rightarrow \eta e^{+} v_{e}\right)$ & $(2.323 \pm 0.063 \pm 0.063) \%$ & $f_{+}^{D_{s}^{+} \rightarrow \eta}(0)\left|V_{c s}\right|$ & $0.4455 \pm 0.0053 \pm 0.0044$ \\
$\mathscr{B}\left(D_{s}^{+} \rightarrow \eta^{\prime} e^{+} v_{e}\right)$ & $(0.824 \pm 0.073 \pm 0.027) \%$ & $f_{+}^{D_{s}^{+} \rightarrow \eta^{\prime}}(0)\left|V_{c s}\right|$ & $0.477 \pm 0.049 \pm 0.011$ \\
\hline
\end{tabular}

The ratio of the decay rates for semileptonic $D$ decays to pseudoscalar mesons with muon or with electron can be used for test of LFU. Such studies have been performed at BESIII, yielding $\frac{\Gamma\left(D^{0} \rightarrow K^{-} \mu^{+} v_{\mu}\right)}{\Gamma\left(D^{0} \rightarrow K^{-} e^{+} v_{e}\right)}=0.974 \pm 0.014, \frac{\Gamma\left(D^{+} \rightarrow \bar{K}^{0} \mu^{+} v_{\mu}\right)}{\Gamma\left(D^{+} \rightarrow \bar{K}^{0} e^{+} v_{e}\right)}=0.988 \pm 0.033, \frac{\Gamma\left(D^{0} \rightarrow \pi^{-} \mu^{+} v_{\mu}\right)}{\Gamma\left(D^{0} \rightarrow \pi^{-} e^{+} v_{e}\right)}=0.922 \pm 0.037$, and $\frac{\Gamma\left(D^{+} \rightarrow \pi^{0} \mu^{+} v_{\mu}\right)}{\Gamma\left(D^{+} \rightarrow \pi^{0} e^{+} v_{e}\right)}=0.964 \pm 0.045[15,19,20]$, these are all consistent with the SM predictions within $2 \sigma$ [21]. One may wish to look at the ratios in different $q^{2}$ intervals which give us better sensitivity to potential LFU violation effect. Figure. 4 shows the results for $D^{0} \rightarrow K^{-} \ell^{+} v_{\ell}$ decays, no significant deviation is observed.

The situation is more complex in the case of semileptonic $D$ decays to vector mesons with the presence of extra polarization vectors. Considering the case of $D^{+} \rightarrow \bar{K}^{* 0} e^{+} v_{e}$ [14], the decay rate is described by three extra angular variables in addition to $q^{2}$, including the angle between the $\pi$ and the $D$ direction in the $K \pi$ rest frame $\left(\theta_{K}\right)$, the angle between the $v_{e}$ and the $D$ direction in the $e v_{e}$ rest frame $\left(\theta_{e}\right)$, and the angle between the two decay planes $(\chi)$ as illustrated in Fig. 5. The BF of the $\bar{K}^{* 0}$ contribution and the extracted form factor ratios are summarized in Table 2.

Similar analyses are also performed for $D^{0} \rightarrow \bar{K}^{0} \pi^{-} e^{+} v_{e}[22], D^{+} \rightarrow \omega e^{+} v_{e}[23], D \rightarrow$ 


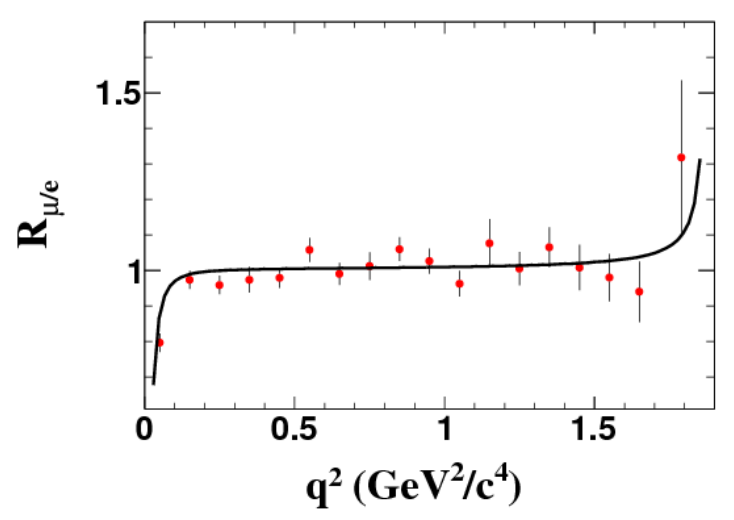

Figure 4: Test for LFU in different $q^{2}$ intervals, where the points with error bars are data and the solid curves are the SM predictions.
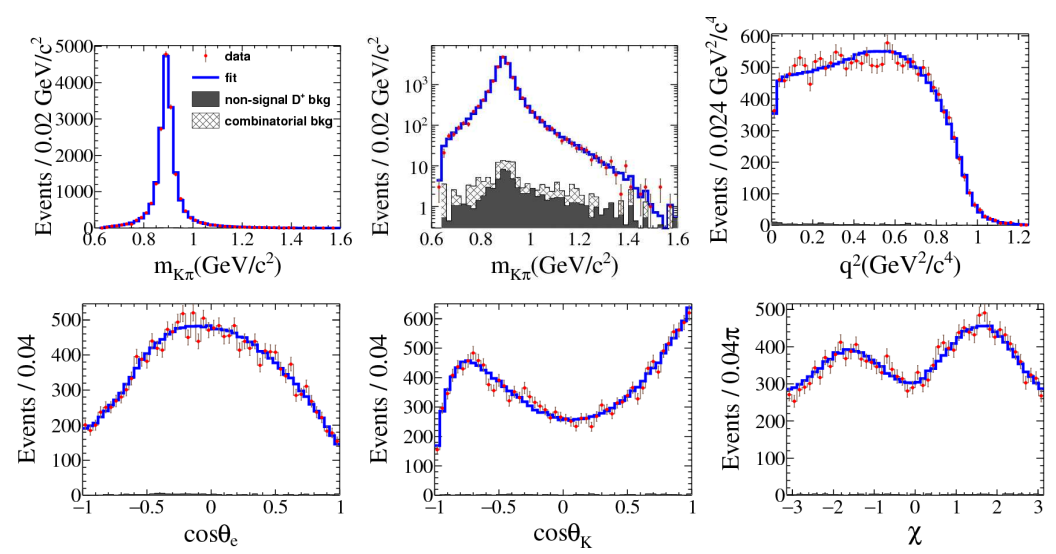

Figure 5: Projections of the fit to the five kinematic variables.

$\pi \pi e^{+} v_{e}$ [24] and $D_{s}^{+} \rightarrow K^{* 0} e^{+} v_{e}$ [18] at BESIII and the results are summarized in Table 2. We also notice that a significant $\mathrm{S}$-wave contribution is observed for $f_{0}(500)$ in $D^{+} \rightarrow \pi^{-} \pi^{+} e^{+} v_{e}$ with $\mathscr{B}\left(D^{+} \rightarrow f_{0}(500) e^{+} v_{e}, f_{0}(500) \rightarrow \pi^{+} \pi^{-}\right)=(0.630 \pm 0.043 \pm 0.032) \times 10^{-3}$, while no evidence for $D^{+} \rightarrow f_{0}(980) e^{+} v_{e}$ is observed. The $\mathrm{U}$-spin symmetry is also found to be conserved with $\frac{r_{V}^{D_{s}^{+} \rightarrow K^{* 0}}}{r_{V}^{D^{+} \rightarrow \rho^{0}}}=1.13 \pm 0.26 \pm 0.11, \frac{r_{2}^{D_{s}^{+} \rightarrow K^{* 0}}}{r_{2}^{D^{+} \rightarrow \rho^{0}}}=0.93 \pm 0.36 \pm 0.10$.

Table 2: The measured BFs and form factor ratios for some of the $D$ meson decays to vector mesons.

\begin{tabular}{lccc}
\hline Decay & BF & $r_{V}$ & $r_{2}$ \\
\hline$D^{+} \rightarrow \bar{K}^{* 0} e^{+} v_{e}$ & $(3.54 \pm 0.03 \pm 0.08) \%$ & $1.41 \pm 0.06 \pm 0.01$ & $0.79 \pm 0.04 \pm 0.01$ \\
$D^{0} \rightarrow \bar{K}^{*-} e^{+} v_{e}$ & $(1.36 \pm 0.03 \pm 0.03) \%$ & $1.46 \pm 0.07 \pm 0.02$ & $0.67 \pm 0.06 \pm 0.01$ \\
$D^{+} \rightarrow \omega e^{+} v_{e}$ & $(1.63 \pm 0.11 \pm 0.08) \times 10^{-3}$ & $1.24 \pm 0.09 \pm 0.06$ & $1.06 \pm 0.15 \pm 0.05$ \\
$D^{0} \rightarrow \rho^{-} e^{+} v_{e}$ & $(1.45 \pm 0.05 \pm 0.04) \times 10^{-3}$ & $1.70 \pm 0.08 \pm 0.05$ & $0.85 \pm 0.06 \pm 0.04$ \\
$D^{+} \rightarrow \rho^{0} e^{+} v_{e}$ & $(1.86 \pm 0.07 \pm 0.06) \times 10^{-3}$ & $1.70 \pm 0.08 \pm 0.05$ & $0.85 \pm 0.06 \pm 0.04$ \\
$D_{s}^{+} \rightarrow K^{* 0} e^{+} v_{e}$ & $(2.37 \pm 0.26 \pm 0.20) \times 10^{-3}$ & $1.67 \pm 0.34 \pm 0.16$ & $0.77 \pm 0.28 \pm 0.07$ \\
\hline
\end{tabular}

It is also interesting to search for semileptonic $D$ decays to scalar mesons which may help us 
understand the internal structure of the light scalar mesons. BESIII has recently searched for $D \rightarrow$ $a_{0}(980) e^{+} v_{e}$ [25], where $D^{0} \rightarrow a_{0}(980)^{-} e^{+} v_{e}$ is observed for the first time with $6.4 \sigma$ significance. The $\mathrm{BF}$ is measured to be $\mathscr{B}\left(D^{0} \rightarrow a_{0}(980)^{-} e^{+} v_{e}, a_{0}(980)^{-} \rightarrow \eta \pi^{-}\right)=\left(1.33_{-0.29}^{+0.33} \pm 0.09\right) \times$ $10^{-4}$. The significance for $D^{+} \rightarrow a_{0}(980)^{0} e^{+} v_{e}$ is $2.9 \sigma$ with $\mathscr{B}\left(D^{+} \rightarrow a_{0}(980)^{0} e^{+} v_{e}, a_{0}(980)^{0} \rightarrow\right.$ $\left.\eta \pi^{0}\right)=\left(1.66_{-0.66}^{+0.81} \pm 0.11\right) \times 10^{-4}$, and less than $3.0 \times 10^{-4}$ at $90 \%$ confidence level. Ref. [26] proposed a model-independent method to study the nature of light scalar mesons with

$$
R=\frac{\mathscr{B}\left(D^{+} \rightarrow f_{0}(980) e^{+} v_{e}\right)+\mathscr{B}\left(D^{+} \rightarrow f_{0}(500) e^{+} v_{e}\right)}{\mathscr{B}\left(D^{+} \rightarrow a_{0}(980)^{0} e^{+} v_{e}\right)} .
$$

$R$ is estimated to be $1.0 \pm 0.3$ for two-quark description of these scalar mesons, and $3.0 \pm 0.9$ for tetraquark description. With BESIII's results we have $R>2.7$ at $90 \%$ confidence level, which favors the tetraquark description.

Recently, BESIII has also observed semileptonic $D^{+}$decays to axial-vector meson $\bar{K}_{1}(1270)^{0}$ [27], with the statistical significance $>10 \sigma$ (See Fig 6). The BF of this decay is determined to be $\mathscr{B}\left[D^{+} \rightarrow \bar{K}_{1}(1270)^{0} e^{+} v_{e}\right]=\left(2.30 \pm 0.26_{\text {stat. }} \pm 0.18_{\text {syst. }} \pm 0.25_{\text {external }}\right) \times 10^{-3}$. Our result indicates $\theta_{K_{1}} \sim 33^{\circ}$ or $57^{\circ}$ and opens up opportunity to precisely study the nature of $\bar{K}_{1}(1270)$.
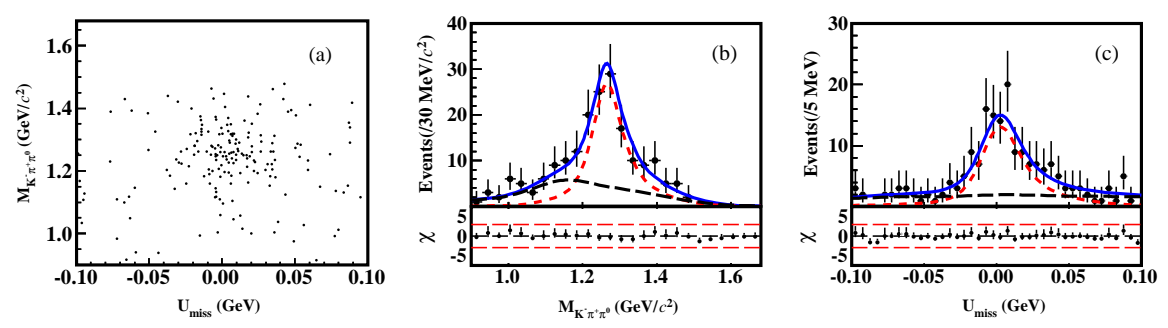

Figure 6: (a) The $M_{K^{-} \pi^{+} \pi^{0}}$ vs. $U_{\text {miss }}$ distribution of the SL candidate events and (b,c) the projections to $M_{K^{-} \pi^{+} \pi^{0}}$ and $U_{\text {miss }}$, respectively, with the residual $\chi$ distributions of the 2-D fit. Dots with error bars are data. Blue solid, red and black dashed curves are the fit result, the fitted signal and the fitted background, respectively.

\section{Summary}

In conclusion, BESIII has reported the most precise measurements of the decay constants of the leptonic $D_{(s)}$ decays, the hadronic form factors of semileptonic $D$ decays, and the CKM matrix elements $\left|V_{c s(d)}\right|$. Meanwhile, LFU tests with these decays have been performed and no evidence of violation is found. Searches for charm semileptonic decays to scalar mesons were performed and our results are in favor of the tetraquark description of $a_{0}(980), f_{0}(500)$ and $f_{0}(980)$. The semileptonic $D$ to axial-vector meson $D^{+} \rightarrow \bar{K}_{1}(1270)^{0} e^{+} v_{e}$ is observed for the first time. With more data coming from BESIII, experimental studies of leptonic and semileptonic $D_{(s)}$ decays will be further improved in the future.

\section{References}

[1] M. Tanabashi et al. (Particle Data Group), Phys. Rev. D98, 030001 (2018). 
[2] M. Ablikim et al. (BESIII Collaboration), Nucl. Instr. Meth. A614, 345 (2010).

[3] M. Ablikim et al. (BESIII Collaboration), Phys. Rev. Lett. 122, 071802 (2019).

[4] A. Bazavov et al. (Fermilab Lattice and MILC Collaborations), Phys. Rev. D98, 074512 (2018).

[5] N. Carrasco et al. (ETM Collaboration), Phys. Rev. D91, 054507 (2015).

[6] J. P. Alexander et al. (CLEO Collaboration), Phys. Rev. D79, 052001 (2009).

[7] P. del Amo Sanchez et al. (BaBar Collaboration), Phys. Rev. D82, 091103 (2010).

[8] A. Zupanc et al. (Belle Collaboration), JHEP 09, 139 (2013).

[9] M. Ablikim et al. (BESIII Collaboration), Phys. Rev. D94, 072004 (2016).

[10] M. Ablikim et al. (BESIII Collaboration), arXiv: 1908.08877.

[11] M. Ablikim et al. (BESIII Collaboration), Phys. Rev. D89, 051104 (2014).

[12] M. Ablikim et al. (BESIII Collaboration), Phys. Rev. D92, 072012 (2015).

[13] M. Ablikim et al. (BESIII Collaboration), Phys. Rev. D96, 012002 (2017).

[14] M. Ablikim et al. (BESIII Collaboration), Phys. Rev. D94, 032001 (2016).

[15] M. Ablikim et al. (BESIII Collaboration), Phys. Rev. Lett. 122, 011804 (2019).

[16] M. Ablikim et al. (BESIII Collaboration), Phys. Rev. D97, 092009 (2018).

[17] M. Ablikim et al. (BESIII Collaboration), Phys. Rev. Lett. 122, 121801 (2019).

[18] M. Ablikim et al. (BESIII Collaboration), Phys. Rev. Lett. 122, 061801 (2019).

[19] M. Ablikim et al. (BESIII Collaboration), Eur. Phys. J. C76, 369 (2016).

[20] M. Ablikim et al. (BESIII Collaboration), Phys. Rev. Lett. 121, 171803 (2018).

[21] L. Riggio, G. Salerno and S. Simula, Eur. Phys. J. C78, 501 (2018).

[22] M. Ablikim et al. (BESIII Collaboration), Phys. Rev. D99, 011103 (2019).

[23] M. Ablikim et al. (BESIII Collaboration), Phys. Rev. D92, 071101 (2015).

[24] M. Ablikim et al. (BESIII Collaboration), Phys. Rev. Lett. 122, 062001 (2019).

[25] M. Ablikim et al. (BESIII Collaboration), Phys. Rev. Lett. 121, 081802 (2018).

[26] W. Wang and C.-D. Lu, Phys. Rev. D82, 034016 (2010).

[27] M. Ablikim et al. (BESIII Collaboration), arXiv: 1907.11370. 